\title{
The Study on In-City Capacity Affected by Pedestrian Crossing
}

\author{
Chang-jiang Zheng, ${ }^{1}$ Rui He, ${ }^{1}$ Xia Wan, ${ }^{2}$ and Chen Wang ${ }^{1,3}$ \\ ${ }^{1}$ School of Civil Engineering and Transportation, Hohai University, Nanjing, Jiangsu 210098, China \\ ${ }^{2}$ Department of Civil and Environmental Engineering, University of Wisconsin-Madison, Madison, WI 53706, USA \\ ${ }^{3}$ Jiangsu Transportation Institute, Nanjing, Jiangsu 210098, China
}

Correspondence should be addressed to Chang-jiang Zheng; zhenghhu@sina.com

Received 16 March 2016; Revised 2 May 2016; Accepted 11 May 2016

Academic Editor: Yongjun Shen

Copyright (C) 2016 Chang-jiang Zheng et al. This is an open access article distributed under the Creative Commons Attribution License, which permits unrestricted use, distribution, and reproduction in any medium, provided the original work is properly cited.

Currently, the urban road traffic congestion is serious and the traffic accident is happening at a high frequency; thus it has not satisfied the travel needs of security and affects the quality of urban trips. In order to effectively relieve the confliction of people and motor vehicle, to make sure of the safety of pedestrians crossing the road, and to improve the capacity of urban roads, this passage focuses on studying the influence of pedestrians crossing the roads on the capacity of urban roads in three pedestrian crossing approaches including freely crossing the street, uncontrolled crossing of the pedestrian crosswalk, and controlled crossing of the pedestrian crosswalk. Firstly, it confirms the general formula of the road capacity when pedestrians are crossing the road based on three preassumptions, combined with the survey data, and then constructs the empirical mathematical model of pedestrian crossing on the capacity impact. Lastly, it takes the step of case calculation and simulation evaluation and calculates errors between them, finding that the error between the model calculation and software simulation is small. The efficiency of the model is validated and improved.

\section{Introduction}

With the rapid development of the social economy and urbanization level, the inventory of motor vehicles in our country is increasing sharply. Though the urban road network has been greatly improved, it still cannot keep up with the pace of growth of motor vehicles. And coupled with a shortage of urban land, road network construction cannot be carried out without restrictions. So how to let network come into a fully play, avoid mutual interference of pedestrians and vehicles, and rationally organize and mix traffic prevails based on the existing road infrastructure has become a necessary means to improve urban road capacity and ease traffic pressure [1].

China, as one of the largest developing countries, has a large population amount. With the rapid development of China's urban residents' travel demand, the proportion of mixed traffic on the road made by pedestrians and vehicles is increasing too. Pedestrian crossing caused a great impact on road capacity [2]. At present, the contradiction between slow traffic whose representative is walk and fast traffic whose representative is motor vehicle is getting increasingly serious, and at the same time this contradiction is difficult to change fundamentally in a short time, so pedestrian traffic and machine moving car traffic must share the limited road resources in the future for a long period of time together [3]. In China's urban traffic accidents, more than half of all are related to pedestrians. Due to the low rate of the crossing pedestrian following traffic rules, the proportion of accidents caused by pedestrians illegally crossing street and severity are much higher than those in developed countries. Therefore, further study of the impact of pedestrian crossing on urban road capacity becomes urgent.

\section{Pedestrian Crossing Behavior Description}

According to the relationship between pedestrians and vehicles in space, pedestrian crossing facilities can be divided into two categories [4]: (1) plane crossing facilities: the facilities can make pedestrians cross the road on the road plane; (2) three-dimensional crossing facilities: the facilities can make pedestrians cross the road at higher level or lower level, 
such as pedestrian footbridge and underground pedestrian passageway. This paper focuses on studying the behavior characteristic of pedestrians who use plane crossing facilities [5].

2.1. Free Pedestrian Crossing Behavior Description. Free pedestrian crossing behavior [6] is the behavior that pedestrians cross the street without any crossing facilities and traffic rules. Pedestrians will according to the actual road conditions, vehicle flow, and vehicle space find reasonable gaps between vehicles to achieve their need of crossing streets when they cross street freely. Pedestrians cross streets based on their own needs, and under most circumstances, pedestrians cross streets alone. That proves the phenomena that there are pedestrians all the time, so it is a way of crossing street which is not recognized and with few security.

2.2. No Pedestrian Crossing Signal Control Behavior Description. Crosswalks at uncontrolled pedestrian crossing [7] refer to the pedestrian crosswalk to reach sections of the no signal at the control after the first observation of whether there are vehicles on the road. If there is no vehicle, pedestrians cross the street immediately; if there are vehicles, pedestrians need to judge whether there is a gap for safe pass; if there is, pedestrian will quickly cross the road with caution; if there is not, pedestrians will wait for it until it appears.

2.3. Pedestrian Crossing Signal Control Behavior Description. Controlled crosswalk pedestrian crossing [8] refers to the behavior that pedestrians cross the street at signalized intersections, and when they arrive at destination, if the traffic light is green, pedestrians cross the street directly; if the traffic light turns red, pedestrians need to wait until the light turns green. In most times, the countdown of the traffic light plays a very important role when pedestrians cross streets. When the time of red light is beyond pedestrians' tolerable time, there will be illegal behavior-pedestrians run the red light and then pedestrians will grub spaces with vehicles to force through the traffic flow [8].

\section{Pedestrian Impact Modeling on Urban Road Capacity}

\subsection{The Basic Concept Definition Model}

3.1.1. The Impact of Pedestrian Crossing Zone. The impact of pedestrian crossing zone this paper studied [9] was based on the assumption that all pedestrians obey the traffic rulespedestrians cross streets by crosswalks. The essence of the impact of pedestrian crossing zone is an area whose conformation is verified with the different standards of crosswalk. The proportion of impact area is generally regarded as the length by the width of the crosswalk [10]. Figure 1 shows a schematic affected zone.

3.1.2. The Impact of Pedestrian Crossing Time (T). Pedestrian impact time is defined as all vehicles' lost time is caused by pedestrian crossing within one hour [11]. Different pedestrian traffic and street control make large differences in pedestrian

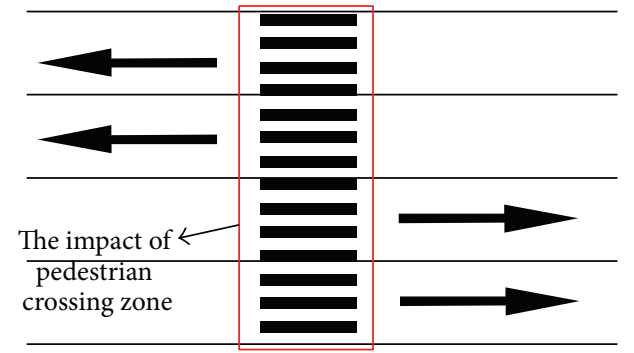

FIGURE 1: A schematic view of the impact of pedestrian crossing zone.

impact time. According to the way pedestrians cross plain roads, the impact of pedestrian crossing time can be divided into three kinds: pedestrian impact free time, no pedestrian crossing signal control affect the time, and pedestrian crossing signal control affect the time.

3.1.3. Section Capacity $C$ under Conditions of Pedestrian Crossing. Is the city's road capacity under the influence of pedestrians crossing the street. The formula is

$$
C=C_{P}(1-k)=C_{P} \frac{3600-T}{3600} .
$$

In these formulas, $C$ is section capacity under the impact of pedestrian crossing $(\mathrm{pcu} / \mathrm{h}) ; C_{P}$ is roads' possible capacity $(\mathrm{pcu} / \mathrm{h}) ; k$ is occupancy rate of pedestrian crossing influencing time; $T$ is pedestrian crossing influencing time.

3.2. Pedestrian Impact Modeling on Urban Road Capacity. According to formulas (1), $C_{P}$ can be obtained from previous studies' results; $T$ can be got from establishing pedestrian's arrival frequency and their relationship and then be translated into the relationship model between pedestrian's arrival frequency and section capacity. This section uses three representative models of SPSS [10] data analysis software in linear models, power model, and quadratic model, to deal with the survey data. Establishing the relationship between pedestrian impact time and pedestrian's arrival frequency in different crossing ways, then we can get the math model about the impact pedestrian crossing exerts on the section capacity.

\subsubsection{Establish the Model of Pedestrian Free Crossing That Has} an Effect on City Capacity. Taking the operability of data collection into account, in the way of pedestrians crossing streets freely, we limited the study objects as pedestrians crossing streets freely and motor vehicles on a $200 \mathrm{~m}$ section of a road. The sample data collection site is a typical one-way road. We use video collection method to record the average traffic condition about pedestrians and motor vehicles in the survey area from 7:00 to 18:00 at five weekdays of crossing.

According to the collected data, we converted five minutes' pedestrian flow rate into 1 hour. Establishing the relationship model about pedestrians average arrival frequency $\lambda$ (person/h) and the impact of time $T$ under the condition of pedestrians crossing streets freely, then we can get a scatter diagram between pedestrian's arrival frequency and effect of 
TABLE 1: Pedestrian crossing under the freedom $\lambda$, and $T$ statistical analysis table.

\begin{tabular}{lcc}
\hline Fitting model & Fitting formula & $R^{2}$ \\
\hline Linear model & $T=0.1969 \lambda+15.781$ & 0.864 \\
Power model & $T=0.2233 \lambda^{0.9932}$ & 0.9237 \\
Quadratic model & $T=-7 \times 10^{-5} \lambda^{2}+0.3156 \lambda-26.557$ & 0.877 \\
\hline
\end{tabular}

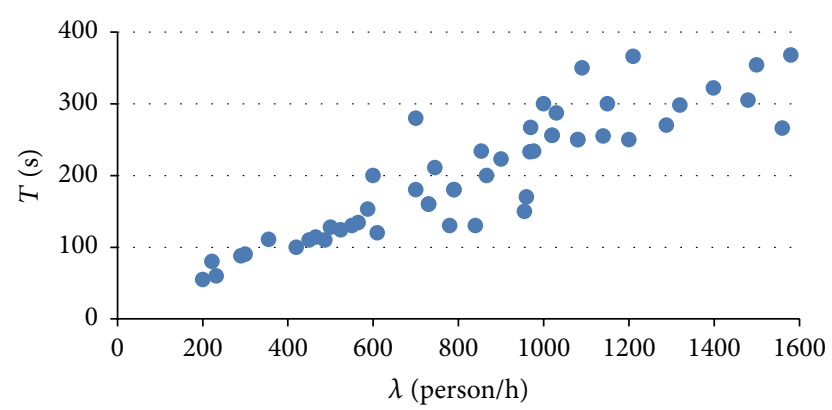

Figure 2: A scatter diagram between $\lambda$ and $T$ under pedestrian free crossing.

time under processing data with SPSS as shown in Figure 2. ( $\lambda$ is the number of pedestrians crossing street successfully hourly.)

From the scatter trend in Figure 2, in free pedestrian crossing condition, there is a positive correlation between pedestrian crossing street impact time and the arrival rate. With the cumulative increase of pedestrian crossing, the impact of pedestrian crossing time is increasing too, and the increasing rate of the two variables keeps balanced roughly. Now we use linear model, power model, and quadratic model to take a regression about the collected data of pedestrian crossing street impact time and the arrival rate. Correlation statistical test results are in Table 1.

From the Table 1 , by comparing various models $R^{2}$, we can know that power model's test number $R^{2}$ is higher than the other two models, so the power model is the best model to describe the relationship between pedestrian arrival rate and influence time under free pedestrian crossing. In summary, we can get the mathematical model:

$$
T=0.2233 \lambda^{0.9932} \text {. }
$$

So, in free pedestrian street conditions, city section capacity empirical formula:

$$
\begin{aligned}
C & =C_{P}(1-k)=C_{P} \frac{3600-T}{3600} \\
& =C_{P}\left(1-\frac{0.2233 \lambda^{0.9932}}{3600}\right) .
\end{aligned}
$$

3.2.2. Crosswalks at Uncontrolled Pedestrian Impact Modeling on Urban Traffic Capacity. The sample data of pedestrian and vehicle at uncontrolled crosswalks were collected in an uncontrolled crosswalk on typical urban road sections. Using video collection method, we can get the average traffic
TABLE 2: Uncontrolled crosswalk, $\lambda$ and $T$ statistical analysis table.

\begin{tabular}{lcc}
\hline Fitting model & Fitting formula & $R^{2}$ \\
\hline Linear model & $T=1.3921 \lambda+76.3742$ & 0.975 \\
Power model & $T=2.6591 \lambda^{0.9145}$ & 0.9849 \\
Quadratic model & $T=6 \times 10^{-6} \lambda^{2}+1.3809 \lambda+80.366$ & 0.9891 \\
\hline
\end{tabular}

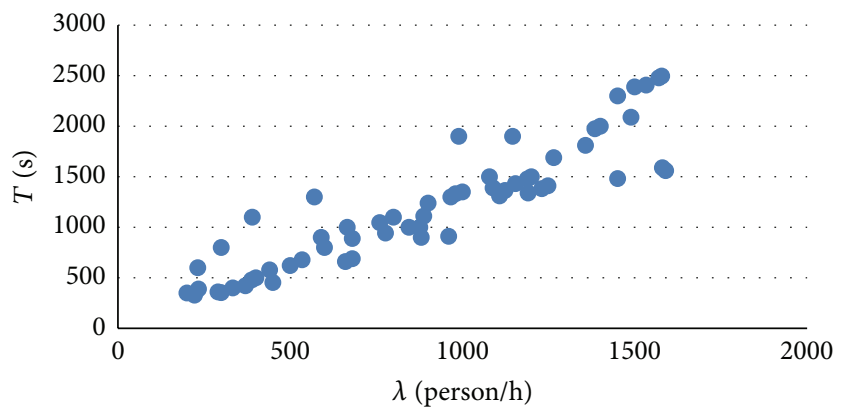

FIgURE 3: Relationship between $\lambda$ and $T$ under uncontrolled crosswalk.

condition about pedestrian and vehicle in the survey area from 7:00 to 18:00 at five weekdays crossing.

According to the collected data, we converted five minutes' pedestrian flow rate into 1 hour. Establishing the relationship model about $\lambda$ and $T$ under an uncontrolled crosswalk, then we can get a scatter diagram with SPSS as shown in Figure 3.

The scatter trend shown in Figure 3 indicated that there is a positive correlation between pedestrian crossing street impact time and the arrival rate in uncontrolled crosswalk condition. With the increase of pedestrian crossing, impact of pedestrian crossing time is increasing too, and the increasing rate of the two variables keeps balanced roughly. Now use linear model, power model, and quadratic model to take a regression about the collected data of pedestrian crossing street impact time and the arrival rate. Correlation statistical test results are in Table 2.

From the Table 2, by comparing various models $R^{2}$, we can know that quadratic model's test number $R^{2}$ is higher than the other two models, so quadratic model is the best model to describe the relationship between pedestrian arrival rate and influence time under uncontrolled crosswalk. In summary, we can get the mathematical model:

$$
T=6 \times 10^{-6} \lambda^{2}+1.3809 \lambda+80.366
$$

Therefore, in the noncontrol crosswalk, pedestrian crossing conditions, traffic capacity of city road experience calculation formula is:

$$
\begin{aligned}
C & =C_{P}(1-k)=C_{P} \frac{3600-T}{3600} \\
& =C_{P}\left(1-\frac{6 \times 10^{-6} \lambda^{2}+1.3809 \lambda+80.366}{3600}\right) .
\end{aligned}
$$


TABLE 3: $\lambda$ and $T$ statistical analysis table of controlled crosswalk.

\begin{tabular}{lcc}
\hline Fitting model & Fitting formula & $R^{2}$ \\
\hline Linear model & $T=0.0567 \lambda+784$ & 0.585 \\
Index model & $T=784.99 e^{7 \times 10^{-5} \lambda}$ & 0.5847 \\
Quadratic model & $T=-10^{-5} \lambda^{2}+0.0754 \lambda+777.31$ & 0.6083 \\
\hline
\end{tabular}

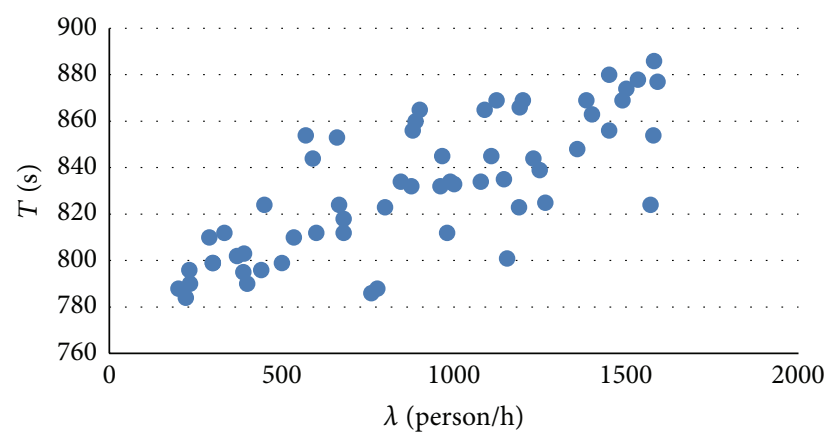

Figure 4: The $\lambda$ and $T$ scatterplot of the controlled crosswalk.

3.2.3. A Controlled Pedestrian Crosswalk at the Effects of Modeling on Urban Traffic Capacity. The sample data of pedestrian crossing and vehicle at controlled crosswalks were collected in a controlled crosswalk on a typical urban road sections. Using video collection method, we can get the average traffic condition about pedestrian and vehicle in the survey area from 7:00 to 18:00 at five weekdays crossing.

According to the collected data, we converted five minutes' pedestrian flow rate into 1 hour's. Establishing the relationship model about pedestrian average arrival frequency $\lambda$ (person/h) and influence of time $T$ under a controlled crosswalk, and then we can get a scatter diagram with SPSS as shown in Figure 4.

As the scatter trend shown in Figure 4 shows, in controlled crosswalk condition, with the cumulative increase of pedestrian crossing, there is a slight increase about the impact of pedestrian crossing. This shows that in the place of controlled signal crosswalks, the amount of pedestrians crossing the street is not the main factor influencing the pedestrian crossing effect time, but controlled by the road lights timing. Now use linear model, power model, and quadratic model to take a regression about the collected data of pedestrian crossing street impact time and the arrival rate. Correlation statistical test results are in Table 3 .

Table 3 shows that by comparing the various models of $R^{2}$ values, the quadratic model's test of $R^{2}$ is higher than the other two models, so using the quadratic model fitting with controlled crosswalk pedestrian crossing condition of the pedestrian crossing pedestrians arrival rate and the influence of time is better. To sum up, in a crosswalk with signal control, crossing pedestrian arrival rate and pedestrian crossing influence time empirical mathematical model as follows:

$$
T=-10^{-5} \lambda^{2}+0.0754 \lambda+777.31 \text {. }
$$

TABLE 4: Experience section capacity calculation under pedestrian freely crossing.

\begin{tabular}{lccccc}
\hline$\lambda($ person $/ \mathrm{h})$ & 400 & 600 & 800 & 1000 & 1200 \\
\hline$T_{\text {free }}(\mathrm{s})$ & 85.75 & 128.28 & 170.70 & 213.05 & 255.35 \\
$C(\mathrm{pcu} / \mathrm{h})$ & 1171 & 1157 & 1143 & 1129 & 1115 \\
\hline
\end{tabular}

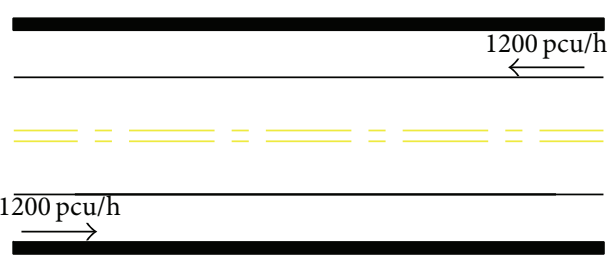

FIGURE 5: Schematic diagram of the pedestrian free crossing road form.

Thus, in controlled crosswalk, under the conditions of pedestrian crossing, urban road capacity empirical formula is

$$
\begin{aligned}
C & =C_{P}(1-k)=C_{P} \frac{3600-T}{3600} \\
& =C_{P}\left(1-\frac{-10^{-5} \lambda^{2}+0.0754 \lambda+777.31}{3600}\right) .
\end{aligned}
$$

3.2.4. Verify the Feasibility. In order to verify the feasibility of the model, we were looking for a typical one-way road, an uncontrolled crosswalk, and a crosswalk to collect sample data on typical urban road sections, and the obtained results are similar to the above conclusion. So three models in which three cases are selected are rather accurate and effective.

\section{Calculation and Simulation Evaluation}

\subsection{Under the Condition of Pedestrian Crossing, Urban Road Traffic Capacity Calculation, and Simulation Evaluation}

4.1.1. Under the Condition of Pedestrian Crossing and Urban Road Traffic Capacity Calculation. In a city road, two-way four lanes, each lane's width is $3.25 \mathrm{~m}$, and the designed speed is $50 \mathrm{~km} / \mathrm{h}$. According to field investigation and related data, every lane's possible capacity is $1200 \mathrm{pcu} / \mathrm{h}$. Now please, respectively, calculate the actual capacity of road section under conditions of the pedestrians flow crossing the street of the entire sections is 400 person $/ \mathrm{h}, 600$ person $/ \mathrm{h}, 800$ person/h, 1000 person/h and 1200 person/h and two-way equal. Road form is shown in Figure 5.

According to the empirical formula (2), we can calculate different influence time $T_{\text {free }}$ of pedestrian flow freely crossing the street at the pedestrian crossing conditions and plug $T_{\text {free }}$ into formula (3) to obtain capacity values of different pedestrian flow, the specific values in Table 4.

4.1.2. The City Road Capacity Simulation Evaluation Effect under the Condition of Pedestrian Crossing. Simulation parameters are selected according to the basic conditions 

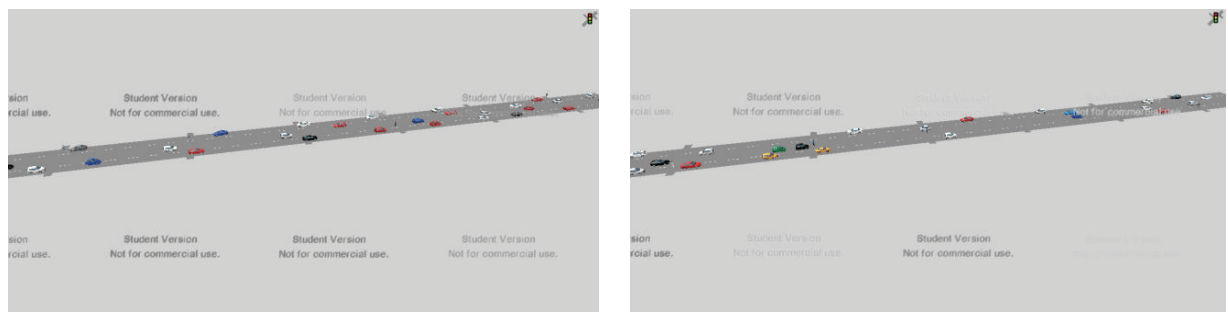

FIGURE 6: The simulation screenshot of the city road traffic capacity under pedestrian free crossing.

TABLE 5: Main parameters of free crossing pedestrian traffic simulation.

\begin{tabular}{lc}
\hline The main parameters & Numerical \\
\hline Simulation time $(\mathrm{s})$ & 600 \\
Lanes & 4 \\
Lane width $(\mathrm{m})$ & 3.25 \\
Possible capacity $(\mathrm{pcu} / \mathrm{h})$ & 1200 \\
Design speed $(\mathrm{km} / \mathrm{h})$ & 50 \\
People crossing the street $($ person $/ \mathrm{h})$ & $400,600,800,1000,1200$
\end{tabular}

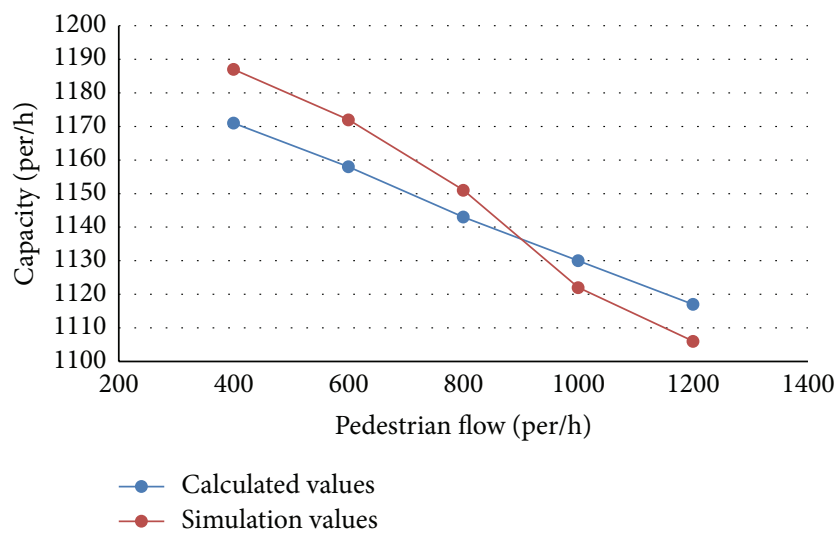

FIGURE 7: Pedestrian crossing traffic capacity under the condition of free comparison chart.

of the research section, and the main parameters of the simulation section are shown in Table 5.

Through the calibration of the simulation parameters, the simulation of the city road traffic capacity under pedestrian free crossing is shown in Figure 6.

By setting "Vehicle travel time" detector, click Run VISSIM software [11]; we can get different simulation values of urban road capacity under conditions of different pedestrian flow freely crossing the street. Thus, the value of road capacity and the simulated result from software output can be got from the model calculation and are shown in Table 6.

According to Table 6, we can obtain the contrast curve of the city road traffic capacity values under the condition that pedestrians cross crosswalk freely and the result is shown in Figure 7.
TABle 6: Pedestrian crossing traffic capacity under the condition of freedom.

\begin{tabular}{lccccc}
\hline $\begin{array}{l}\text { Pedestrian flow } \\
\text { (person/h) }\end{array}$ & 400 & 600 & 800 & 1000 & 1200 \\
\hline $\begin{array}{l}\text { Model calculation value } \\
(\text { pcu/h) }\end{array}$ & 1171 & 1157 & 1143 & 1129 & 1115 \\
$\begin{array}{l}\text { Software simulation } \\
\text { value (pcu/h) }\end{array}$ & 1182 & 1176 & 1158 & 1119 & 1047 \\
\hline
\end{tabular}

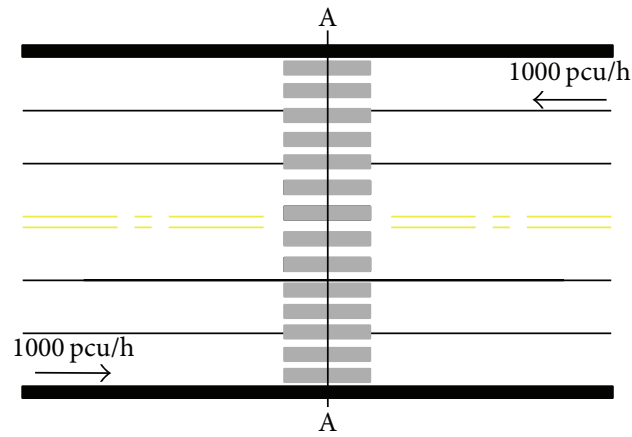

FIGURE 8: Schematic diagram of uncontrolled crosswalk road form.

\subsection{Example Calculation of Urban Road Capacity and Simu- lation Evaluation under No Control of Pedestrian Crossing}

4.2.1. Example Calculation of Urban Road Capacity under No Control of Pedestrian Crossing. In a city road for twoway six lanes, each lane's width is $3.5 \mathrm{~m}$, and designed speed is $40 \mathrm{~km} / \mathrm{h}$. According to field investigation and consulting related data, we can get that each lane's possible capacity is $1000 \mathrm{pcu} / \mathrm{h}$. Setting pedestrian's walking speed is $1.30 \mathrm{~m} / \mathrm{s}$, then, respectively, calculate the actual capacity of sections under conditions of the pedestrians flow crossing the street of the entire sections is 400 person/h, 600 person $/ \mathrm{h}, 800$ person/h, 1000 person/h and 1200 person/h and two-way equal. The implementation of pedestrian crossing behavior in the A-A section, and the section is uncontrolled crosswalk, road form as shown in Figure 8.

According to the empirical formula (4), we can calculate different street pedestrian flow freely cross the street at the pedestrian crossing conditions affect the time $T_{\text {uncontrol}}$, plug $T_{\text {uncontrol }}$ into formula (5) to obtain capacity values of different people, the specific values in Table 7. 

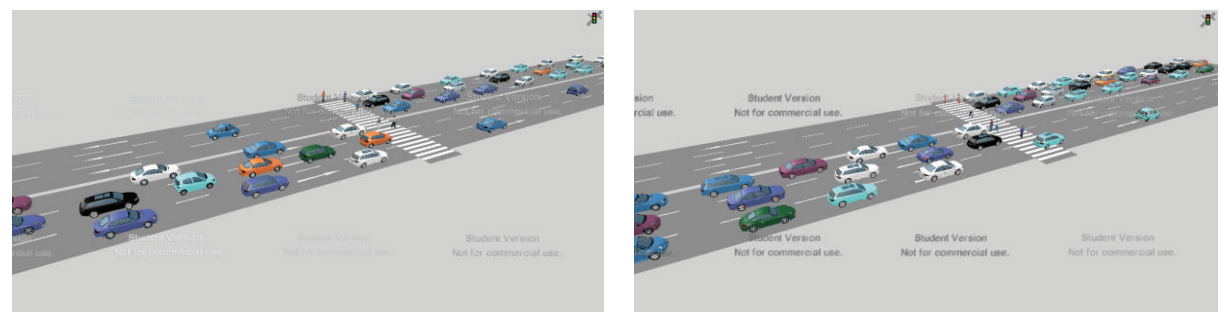

FIGURE 9: Simulation screenshot of uncontrolled crosswalk located under the condition of.

TABLE 7: Experience link capacity control crosswalk pedestrian condition calculation.

\begin{tabular}{lccccc}
\hline$\lambda($ person $/ \mathrm{h})$ & 400 & 600 & 800 & 1000 & 1200 \\
\hline$T_{\text {uncontrol }}(\mathrm{s})$ & 565.24 & 784.86 & 1245.64 & 1483.21 & 1713.65 \\
$C(\mathrm{pcu} / \mathrm{h})$ & 843 & 782 & 654 & 588 & 524 \\
\hline
\end{tabular}

TABLE 8: Main parameters of uncontrolled crosswalk section.

\begin{tabular}{lc}
\hline Main parameters & Numerical \\
\hline Simulation time $(\mathrm{s})$ & 600 \\
Lane number & 6 \\
Lane width $(\mathrm{m})$ & 3.5 \\
Potential capacity $(\mathrm{pcu} / \mathrm{h})$ & 1000 \\
Design speed $(\mathrm{km} / \mathrm{h})$ & 40 \\
Pedestrian speed $(\mathrm{m} / \mathrm{s})$ & 1.3 \\
Crossing number $($ person $/ \mathrm{h})$ & $400,600,800,1000,1200$ \\
\hline
\end{tabular}

4.2.2. The Simulated Evaluation of Uncontrolled Crosswalk Pedestrian Crossing under the Condition of City Road Traffic Capacity. Simulation parameters are selected according to the basic conditions of the research section, and the main parameters of the section are shown in Table 8.

Through the calibration parameters we simulated the traffic capacity of urban road under different pedestrian flow. No pedestrians in the crosswalk crossing control effect are shown in Figure 9 under the city road traffic capacity simulation.

By setting "Vehicle travel time" detector, click Run VISSIM software; we can get different simulation values of urban road capacity under conditions of different pedestrian flow freely crossing the street. Thus, the value of road capacity and the simulated result from software output can be got from the model calculation and are as shown in Table 9.

According to Table 9 data, we can obtain the contrast curve of the city road traffic capacity values under the condition of pedestrian crossing crosswalk without signal control and the result is shown in Figure 10.

\subsection{A Pedestrian Crosswalk Control under the Condition of City Road Traffic Capacity Calculation and Simulation Effect Evaluation}

4.3.1. Pedestrian Crosswalk Control under the Condition of City Road Traffic Capacity Calculation Effect. A city road, two-way four lanes, each lane's width is $3.25 \mathrm{~m}$, and the

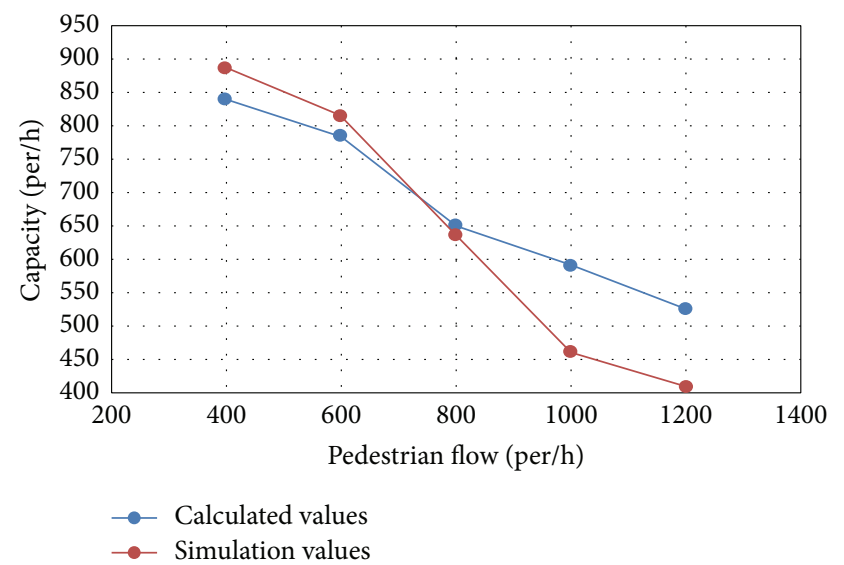

FIGURE 10: Section of uncontrolled crosswalk at pedestrian crossing under the condition of capacity comparison chart.

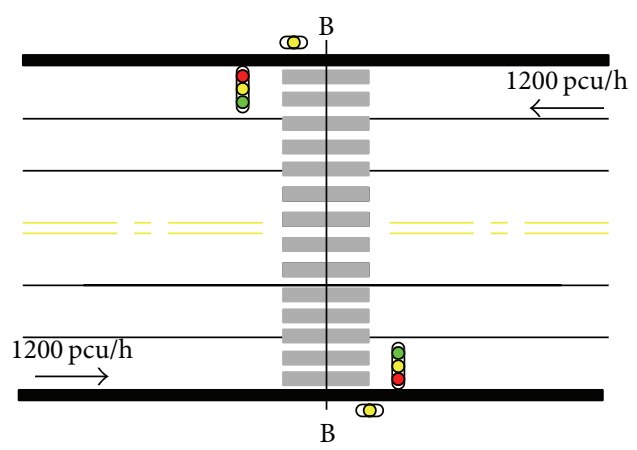

FIGURE 11: A schematic diagram of control crosswalk road form.

designed speed is $50 \mathrm{~km} / \mathrm{h}$. According to field investigation and related data, we can get that every lane's possible capacity is $1200 \mathrm{pcu} / \mathrm{h}$. Setting pedestrian's walking speed is $1.30 \mathrm{~m} / \mathrm{s}$. Pedestrians cross the street in B-B section implementation. The section is a controlled crosswalk, signal cycle's length is $75 \mathrm{~s}$, and the red light lasts $24 \mathrm{~s}$. As investigated in the site, motor vehicle saturated headway distance is $2.05 \mathrm{~s}$. Now please, respectively, calculate the actual capacity of crossing the streets under conditions of the pedestrians flow about 400 persons $/ \mathrm{h}, 600$ persons $/ \mathrm{h}, 800$ persons/h, 1000 persons/h, and 1200 persons/h and two-way equality of the entire sections. Road form is shown in Figure 11.

According to the empirical formula (6), pedestrian crossing influence time $T_{\text {control }}$ can be calculated in different 

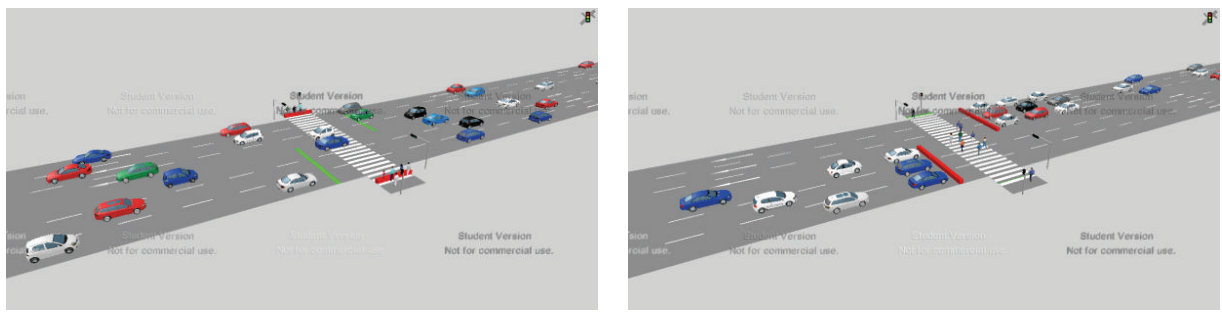

FIGURE 12: Simulation screenshot of crosswalk capacity under the condition of pedestrian crossing.

TABLE 9: Road crossing control under the condition of pedestrian crossing traffic capacity.

\begin{tabular}{lccccc}
\hline $\begin{array}{l}\text { Crossing number } \\
\text { (person/h) }\end{array}$ & 400 & 600 & 800 & 1000 & 1200 \\
\hline $\begin{array}{l}\text { Model calculation value } \\
\text { (pcu/h) }\end{array}$ & 843 & 782 & 654 & 588 & 524 \\
$\begin{array}{l}\text { Software simulation } \\
\text { value }(\mathrm{pcu} / \mathrm{h})\end{array}$ & 884 & 816 & 634 & 464 & 408 \\
\hline
\end{tabular}

TABLE 10: Section capacity experience value under the conditions of pedestrian crossing the controlled crosswalk.

\begin{tabular}{lccccc}
\hline$\lambda($ person $/ \mathrm{h})$ & 400 & 600 & 800 & 1000 & 1200 \\
\hline$T_{\text {control }}(\mathrm{s})$ & 805.87 & 818.95 & 831.23 & 842.71 & 853.39 \\
$C(\mathrm{pcu} / \mathrm{h})$ & 931 & 927 & 923 & 919 & 916 \\
\hline
\end{tabular}

TABLE 11: The main parameter to control the crosswalk traffic simulation.

\begin{tabular}{lc}
\hline Main parameters & Value \\
\hline Simulation time (s) & 600 \\
Lane number (per) & 6 \\
Lane width (m) & 3.25 \\
Potential capacity (pcu/h) & 1200 \\
Signal cycle length (s) & 75 \\
Red time (s) & 24 \\
Design speed $(\mathrm{km} / \mathrm{h})$ & 50 \\
People crossing the street (person/h) & $400,600,800,1000,1200$ \\
\hline
\end{tabular}

pedestrian traffic at uncontrolled crosswalk; $T_{\text {control }}$ will be substituted into formula (7) obtaining experience values of capacity under different flow; specific values are shown in Table 10.

\subsubsection{Simulation Evaluation of Influencing the Urban Road} Capacity under the Conditions of Pedestrian Crossing the Controlled Crosswalk. The main parameters of the simulation section are the basic information of the road, and the main parameters of the section are shown in Table 11.

Through the calibration parameters, the traffic capacity of urban road under different pedestrian flow is simulated. The simulation of city road traffic capacity under the influence of pedestrian crossing the controlled crosswalk is shown in Figure 12.
TABLE 12: Section capacity under the conditions of pedestrian crossing the controlled crosswalk.

\begin{tabular}{lccccc}
\hline $\begin{array}{l}\text { Pedestrian flow } \\
\text { (person/h) }\end{array}$ & 400 & 600 & 800 & 1000 & 1200 \\
\hline $\begin{array}{l}\text { Model calculation value } \\
\text { (pcu/h) }\end{array}$ & 931 & 927 & 923 & 919 & 916 \\
$\begin{array}{l}\text { Software simulation } \\
\text { value }(\mathrm{pcu} / \mathrm{h})\end{array}$ & 976 & 958 & 946 & 936 & 926 \\
\hline
\end{tabular}

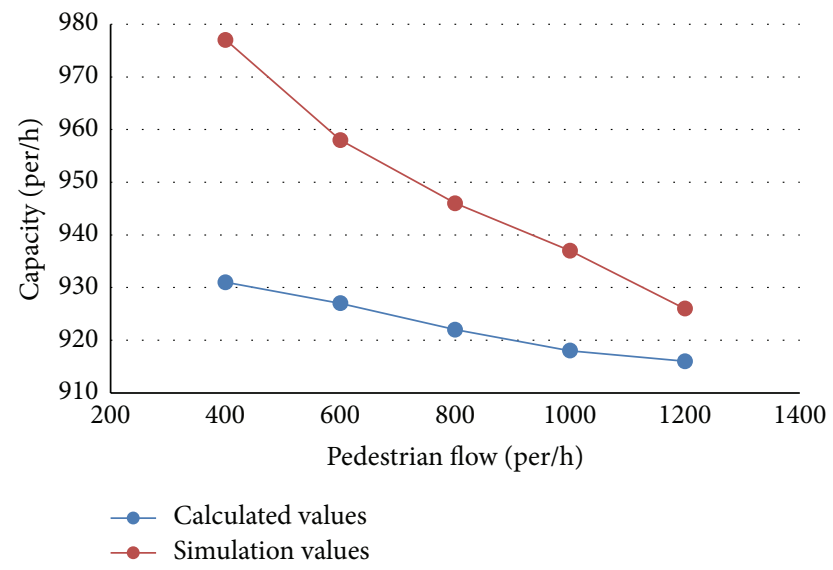

FIGURE 13: Section capacity comparison chart under the conditions of pedestrian crossing the controlled crosswalk.

By setting "Vehicle travel time" detector, click Run VISSIM software; we can get different simulation values of urban road capacity under conditions of different pedestrian flow freely crossing the street. Thus, the value of road capacity and the simulated result from software output can be got from the model calculation and are as shown in Table 12.

According to Table 12, we can obtain the curve contrasting the traffic capacity values of city road under the condition of crossing pedestrian at crosswalks without signal controlling and the result is shown in Figure 13.

\section{Conclusion}

According to contrasting and analyzing the model calculated value and simulated value, and calculating errors between them, the efficiency of the model is validated and improved.

(1) Under the condition of pedestrian crossing freely, the error between the model calculation and software simulation 
is small. When the pedestrian flow is less than 1000 people per hour, the error between the model calculation and the software simulation results is within $2 \%$, so the capacity of the fold reduction rate calculated values got is in line with the actual capacity of the road roughly; when the pedestrian flow is greater than 1000 people per hour, the capacity of the fold reduction rate came from software simulation which is much higher than that coming from the model calculated, which indicated that the model cannot accurately calculate the road capacity. Also as the Figure 7 shows, under the condition of pedestrian crossing freely city, the road capacity of urban section is greatly related to the amount of pedestrian flow: the flow increases and road capacity decreases.

(2) Under the condition of uncontrolled crosswalk pedestrian crossing freely, when the traffic is less than $800 / \mathrm{h}$, the error between the model calculation and the software simulation results is small and within 5\%, so the model calculation value is in line with the actual capacity of the road roughly. Also as Figure 11 shows, city road capacity is greatly related to pedestrian traffic: pedestrian traffic increases and road traffic capacity decreases quickly.

(3) Under the condition of uncontrolled crosswalk pedestrian crossing freely, the error between the model calculation and the software simulation is small, and the error between the calculated value and the simulation value under different pedestrian flow conditions is within $5 \%$, so the model calculation value is in line with the actual capacity of the road roughly. Also as Figure 13 shows, city road capacity is little related to pedestrian traffic in the crosswalk with signals controlling; in a certain range of pedestrian traffic, traffic capacity of city road does not reduce with the increase of pedestrians but mainly depend on the signal timing phase.

\section{Competing Interests}

The authors declare that they have no competing interests.

\section{References}

[1] X. Sheng, Study on Urban Road Traffic Management Impact on Its Capacity, Huazhong University of Science and Technology, 2008.

[2] Transportation Research Board, Highway Capacity Manual 2000, National Research Council, Washington, DC, USA, 2000.

[3] G. A. O. Liping, Pedestrian and Vehicle Conflict Analysis and Modeling Delays about Crosswalk Urban Road Environment, Beijing Jiaotong University, Beijing, China, 2010.

[4] C. A. O. Xiaokui, D. U. Renbing, and P. E. I. Yulong, "The reasonable intervals of road facilities on pedestrian crossing," Transport Information and Safety, vol. 2, pp. 24-27, 2010.

[5] A. K. Gupta, A study on pedestrian walking behavior, University of Delaware, Newark, DE, USA, 2005.

[6] F. Shumin and P. Yulong, "Study of pedestrian crossing delay," Journal of Harbin Institute of Technology, vol. 4, pp. 613-616, 2007.

[7] Y. Xiaoguang, L. Yunteng, and Y. Meiting, "No pedestrian crossing signal control sections of the applicability of the way," Journal of Tongji University (Natural Science), vol. 11, pp. 14661496, 2007.
[8] H. E. Shanglu, C. Zheng, X. Guo et al., "The design on the system of the sections inductive signal controlled pedestrian crossing," Transport Information and Safety, vol. 3, pp. 110-117, 2012.

[9] L. Jian, Y. Qiong, and Y. Dong-Lei, "Pedestrian crossing facilities at reasonable intervals," Traffic and Transportation Engineering, vol. 2, no. 4, pp. 63-67, 2002.

[10] P. Xia, S. Jie, Z. Ruodong et al., "Data analysis and SPSS software applications," Jilin Medical College, vol. 3, pp. 145-147, 2005.

[11] Z. Lidong and W. Yinglong, "Situation analysis of traffic simulation,” Computer Simulation, vol. 6, pp. 255-258, 2006. 


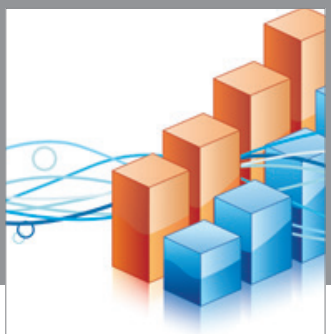

Advances in

Operations Research

vatem alat4

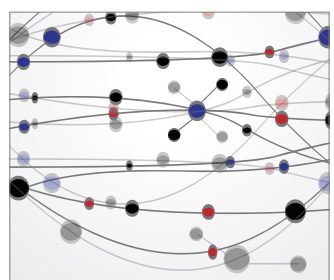

\section{The Scientific} World Journal
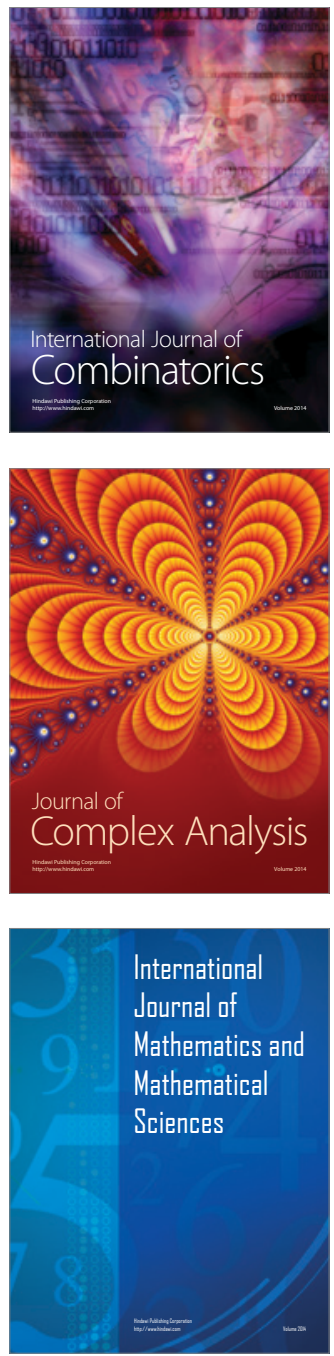
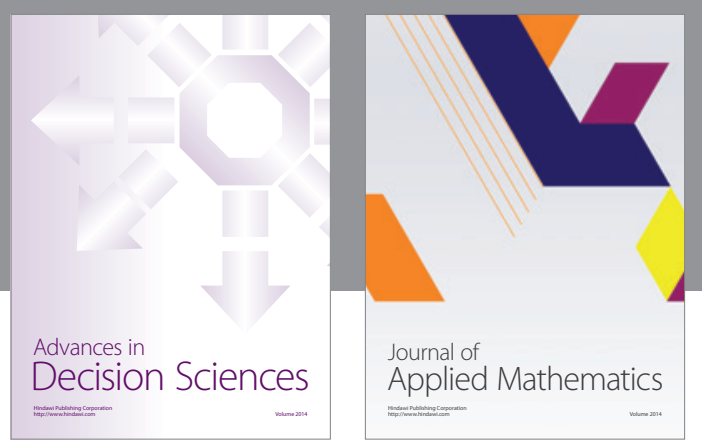

Algebra

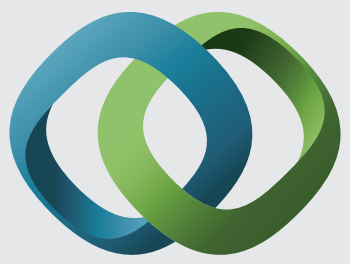

\section{Hindawi}

Submit your manuscripts at

http://www.hindawi.com
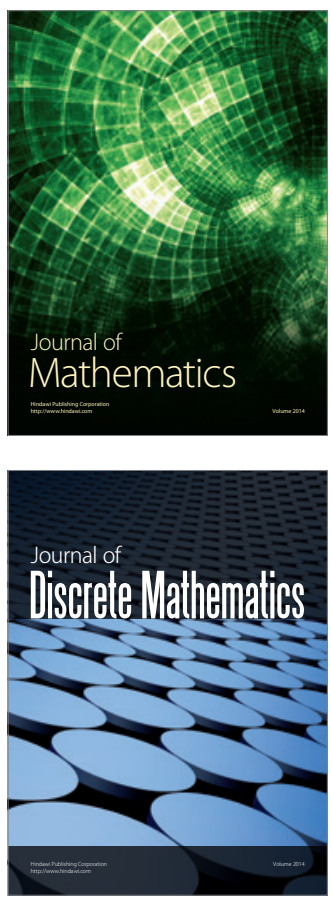

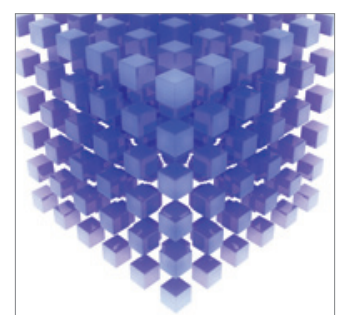

Mathematical Problems in Engineering
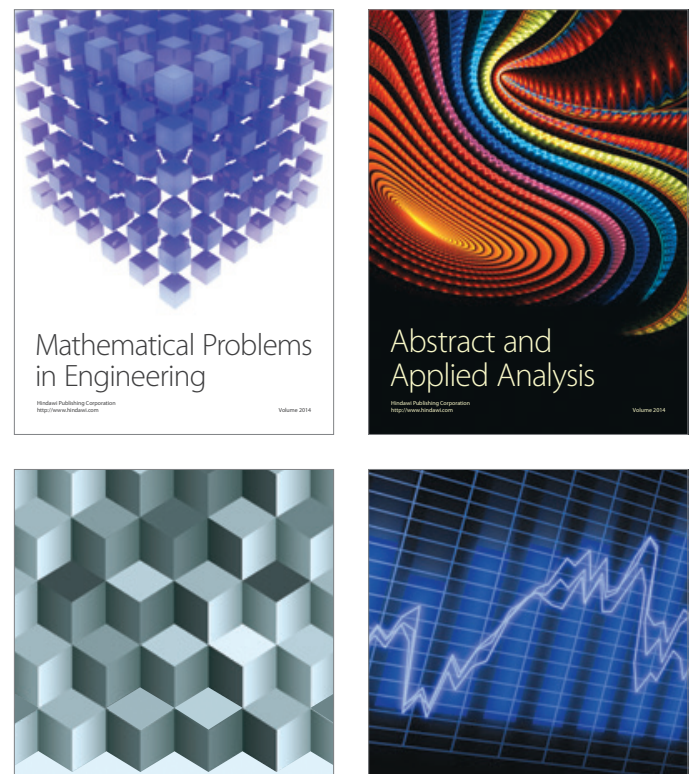

Journal of

Function Spaces

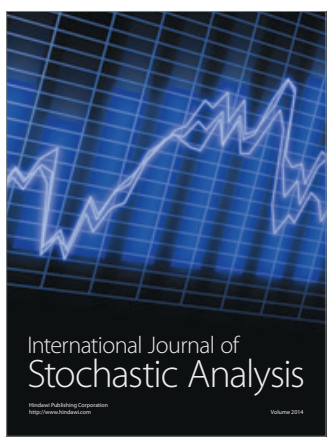

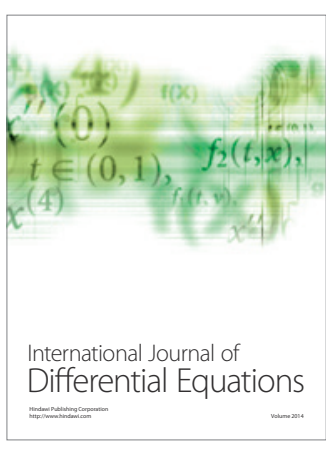
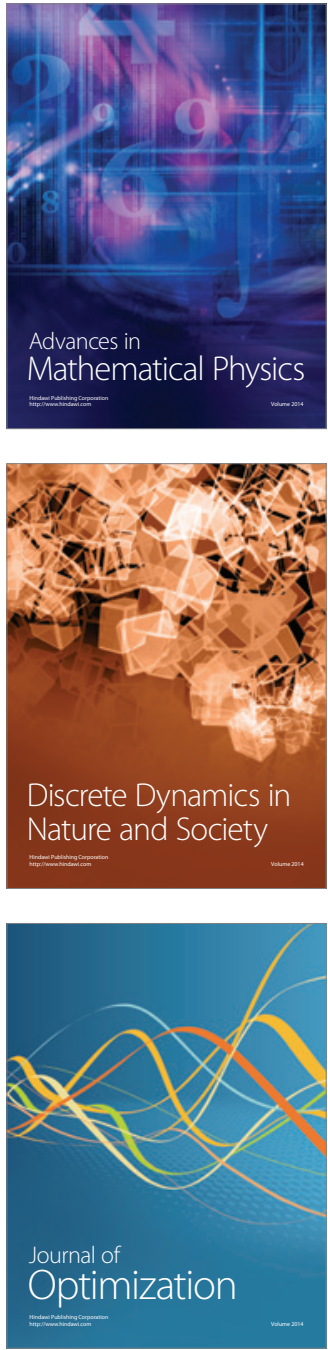